Article

\title{
On Some Inequalities Involving Liouville-Caputo Fractional Derivatives and Applications to Special Means of Real Numbers
}

\author{
Bessem Samet ${ }^{1,2}$ and Hassen Aydi ${ }^{3, *(\mathbb{D}}$ \\ 1 Nonlinear Analysis Research Group, Ton Duc Thang University, Ho Chi Minh City, Vietnam; \\ bessem.samet@tdtu.edu.vn \\ 2 Faculty of Mathematics and Statistics, Ton Duc Thang University, Ho Chi Minh City, Vietnam \\ 3 Department of Mathematics, College of Education in Jubail, Imam Abdulrahman Bin Faisal University, \\ P.O. Box 12020, Jubail 31961, Saudi Arabia \\ * Correspondence: hmaydi@iau.edu.sa
}

Received: 3 September 2018; Accepted: 2 October 2018; Published: 8 October 2018

check for updates

\begin{abstract}
We are concerned with the class of functions $f \in C^{1}([a, b] ; \mathbb{R}), a, b \in \mathbb{R}, a<b$, such that $\left|{ }^{c} D_{a}^{\alpha} f\right|$ is convex or $\left|{ }^{c} D_{b}^{\alpha} f\right|$ is convex, where $0<\alpha<1,{ }^{c} D_{a}^{\alpha} f$ is the left-side Liouville-Caputo fractional derivative of order $\alpha$ of $f$ and ${ }^{c} D_{b}^{\alpha} f$ is the right-side Liouville-Caputo fractional derivative of order $\alpha$ of $f$. Some extensions of Dragomir-Agarwal inequality to this class of functions are obtained. A parallel development is made for the class of functions $f \in C^{1}([a, b] ; \mathbb{R})$ such that $\left|{ }^{c} D_{a}^{\alpha} f\right|$ is concave or $\left|{ }^{c} D_{b}^{\alpha} f\right|$ is concave. Next, an application to special means of real numbers is provided.
\end{abstract}

Keywords: Liouville-Caputo fractional derivative; convexity; Dragomir-Agarwal inequality

MSC: 35A23; 26A33

\section{Introduction}

Let $0<\alpha<1$ and $a, b \in \mathbb{R}$ be such that $a<b$. We consider the classes of functions

$$
\begin{aligned}
& \operatorname{Conv}_{a}^{\alpha}([a, b] ; \mathbb{R})=\left\{f \in C^{1}([a, b] ; \mathbb{R}):\left|{ }^{c} D_{a}^{\alpha} f\right| \text { is convex }\right\} ; \\
& \operatorname{Conv}_{b}^{\alpha}([a, b] ; \mathbb{R})=\left\{f \in C^{1}([a, b] ; \mathbb{R}):\left|{ }^{c} D_{b}^{\alpha} f\right| \text { is convex }\right\} ; \\
& \operatorname{Conc}_{a}^{\alpha}([a, b] ; \mathbb{R})=\left\{f \in C^{1}([a, b] ; \mathbb{R}):\left|{ }^{c} D_{a}^{\alpha} f\right| \text { is concave }\right\} ; \\
& \operatorname{Conc}_{b}^{\alpha}([a, b] ; \mathbb{R})=\left\{f \in C^{1}([a, b] ; \mathbb{R}):\left|{ }^{c} D_{b}^{\alpha} f\right| \text { is concave }\right\},
\end{aligned}
$$

where ${ }^{c} D_{a}^{\alpha} f$ is the left-side Liouville-Caputo fractional derivative of order $\alpha$ of $f$ and ${ }^{c} D_{b}^{\alpha} f$ is the right-side Liouville-Caputo fractional derivative of order $\alpha$ of $f$. In this paper, we extend Dragomir-Agarwal inequality to the above classes of functions. Next, we provide an application to the special means of real numbers.

Let us mention some motivations for studying the proposed problems. Let $f: I \rightarrow \mathbb{R}$ be a given function, where $I$ is a certain interval of $\mathbb{R}$, and let $a, b \in I$ be such that $a<b$. If $f$ is convex in $I$, then

$$
f\left(\frac{a+b}{2}\right) \leq \frac{1}{b-a} \int_{a}^{b} f(x) d x \leq \frac{f(a)+f(b)}{2} .
$$

Inequality (5) is known in the literature as Hermite-Hadamard's inequality (see [1,2]). Several improvements and extensions of inequality (5) to different types of convexity were established by 
many authors. In this direction, we refer the reader to [2-8] and the references therein. In [9], Dragomir and Agarwal established the following interesting result, which provides an estimate between the difference between the middle and right terms in inequality (5).

Theorem 1 (Dragomir-Agarwal inequality). Let $f: I \rightarrow \mathbb{R}$ be a given function, where $I$ is a certain interval of $\mathbb{R}$, and let $a, b \in I$ be such that $a<b$. If $f$ is differentiable in I and $\left|f^{\prime}\right|$ is convex in $[a, b]$, then

$$
\left|\frac{f(a)+f(b)}{2}-\frac{1}{b-a} \int_{a}^{b} f(x) d x\right| \leq \frac{(b-a)\left(\left|f^{\prime}(a)\right|+\mid f^{\prime}(b)\right)}{8} .
$$

The main idea for proving Theorem 1 is based on the following lemma [9].

Lemma 1. Let $f: I \rightarrow \mathbb{R}$ be a given function, where $I$ is a certain interval of $\mathbb{R}$, and let $a, b \in I$ be such that $a<b$. If $f$ is differentiable in I and $f^{\prime} \in L^{1}(] a, b[; \mathbb{R})$, then

$$
\frac{f(a)+f(b)}{2}-\frac{1}{b-a} \int_{a}^{b} f(x) d x=\frac{b-a}{2} \int_{0}^{1}(1-2 t) f^{\prime}(t a+(1-t) b) d t .
$$

In [10], Pearce and Pečarić extended Theorem 1 to the case when $\left|f^{\prime}\right|$ is concave. Using Lemma 1 and Jensen integral inequality, they obtained the following interesting result.

Theorem 2. Let $f: I \rightarrow \mathbb{R}$ be a given function, where $I$ is a certain interval of $\mathbb{R}$, and let $a, b \in I$ be such that $a<b$. If $f$ is differentiable in $\stackrel{\circ}{I}$ and $\left|f^{\prime}\right|$ is concave in $[a, b]$, then

$$
\left|\frac{f(a)+f(b)}{2}-\frac{1}{b-a} \int_{a}^{b} f(x) d x\right| \leq \frac{b-a}{4}\left|f^{\prime}\left(\frac{a+b}{2}\right)\right| .
$$

Motivated by the above cited works, our aim in this paper is to extend Theorems 1 and 2 to the classes of functions given by (1)-(4).

The rest of the paper is organized as follows. In Section 2, we recall some basic concepts on fractional calculus. In Section 3, we state and prove our main results. In Section 4, an application to special means of real numbers is provided.

\section{Preliminaries}

In this section, we recall some basic notions on fractional calculus. For more details, we refer the reader to $[11,12]$.

First, let us fix $a, b \in \mathbb{R}$ with $a<b$.

Definition 1. The left-side Riemann-Liouville fractional integral of order $\sigma>0$ of a function $f \in C([a, b] ; \mathbb{R})$ is given by

$$
\left(I_{a}^{\sigma} f\right)(x)=\frac{1}{\Gamma(\sigma)} \int_{a}^{x}(x-t)^{\sigma-1} f(t) d t, \quad a<x \leq b,
$$

where $\Gamma$ denotes the Gamma function.

Definition 2. The right-side Riemann-Liouville fractional integral of order $\sigma>0$ of a function $f \in C([a, b] ; \mathbb{R})$ is given by

$$
\left(I_{b}^{\sigma} f\right)(x)=\frac{1}{\Gamma(\sigma)} \int_{x}^{b}(t-x)^{\sigma-1} f(t) d t, \quad a \leq x<b .
$$

Lemma 2. Let $\sigma>0$ and $f \in C([a, b] ; \mathbb{R})$. Then,

$$
\left(I_{a}^{\sigma} f\right)\left(a^{+}\right)=\left(I_{b}^{\sigma} f\right)\left(b^{-}\right)=0,
$$


where

$$
\left(I_{a}^{\sigma} f\right)\left(a^{+}\right)=\lim _{x \rightarrow a^{+}}\left(I_{a}^{\sigma} f\right)(x)
$$

and

$$
\left(I_{b}^{\sigma} f\right)\left(b^{-}\right)=\lim _{x \rightarrow b^{-}}\left(I_{b}^{\sigma} f\right)(x) .
$$

Furthermore, for $\sigma>0$ and $f \in C([a, b] ; \mathbb{R})$, we set

$$
\left(I_{a}^{\sigma} f\right)(a)=\left(I_{a}^{\sigma} f\right)\left(a^{+}\right)=0
$$

and

$$
\left(I_{b}^{\sigma} f\right)(b)=\left(I_{b}^{\sigma} f\right)\left(b^{-}\right)=0 .
$$

Lemma 3. Let $f \in C([a, b] ; \mathbb{R})$. Then,

$$
\lim _{\sigma \rightarrow 0^{+}}\left(I_{a}^{\sigma} f\right)(x)=f(x), \quad a<x \leq b
$$

and

$$
\lim _{\sigma \rightarrow 0^{+}}\left(I_{b}^{\sigma} f\right)(x)=f(x), \quad a \leq x<b .
$$

Lemma 4. Let $\sigma>0$ and $f, g \in C([a, b] ; \mathbb{R})$. Then,

$$
\int_{a}^{b}\left(I_{a}^{\sigma} f\right)(x) g(x) d x=\int_{a}^{b}\left(I_{b}^{\sigma} g\right)(x) f(x) d x .
$$

Definition 3. The left-side Liouville-Caputo fractional derivative of order $0<\alpha<1$ of a function $f \in$ $C^{1}([a, b] ; \mathbb{R})$ is given by

$$
\left({ }^{c} D_{a}^{\alpha} f\right)(x)=\left(I_{a}^{1-\alpha} f^{\prime}\right), \quad a<x \leq b .
$$

Definition 4. The right-side Liouville-Caputo fractional derivative of order $0<\alpha<1$ of a function $f \in$ $C^{1}([a, b] ; \mathbb{R})$ is given by

$$
\left({ }^{c} D_{b}^{\alpha} f\right)(x)=-\left(I_{b}^{1-\alpha} f^{\prime}\right), \quad a<x \leq b .
$$

The following result is an immediate consequence of Lemma 2.

Lemma 5. Let $0<\alpha<1$ and $f \in C^{1}([a, b] ; \mathbb{R})$. Then,

$$
\left({ }^{c} D_{a}^{\alpha} f\right)\left(a^{+}\right)=\left({ }^{c} D_{b}^{\alpha} f\right)\left(b^{-}\right)=0,
$$

where

$$
\left({ }^{c} D_{a}^{\alpha} f\right)\left(a^{+}\right)=\lim _{x \rightarrow a^{+}}\left({ }^{c} D_{a}^{\alpha} f\right)(x)
$$

and

$$
\left({ }^{c} D_{b}^{\alpha} f\right)\left(b^{-}\right)=\lim _{x \rightarrow b^{-}}\left({ }^{c} D_{b}^{\alpha} f\right)(x) .
$$

Furthermore, for $0<\alpha<1$ and $f \in C^{1}([a, b] ; \mathbb{R})$, we set

$$
\left({ }^{c} D_{a}^{\alpha} f\right)(a)=\left({ }^{c} D_{a}^{\alpha} f\right)\left(a^{+}\right)=0,
$$

and

$$
\left({ }^{c} D_{b}^{\alpha} f\right)(b)=\left({ }^{c} D_{b}^{\alpha} f\right)\left(b^{-}\right)=0 .
$$


The following result is an immediate consequence of Lemma 3.

Lemma 6. Let $f \in C^{1}([a, b] ; \mathbb{R})$. Then,

$$
\lim _{\alpha \rightarrow 1^{-}}\left({ }^{c} D_{a}^{\alpha} f\right)(x)=f^{\prime}(x), \quad a<x \leq b
$$

and

$$
\lim _{\alpha \rightarrow 0^{+}}\left({ }^{c} D_{b}^{\alpha} f\right)(x)=-f^{\prime}(x), \quad a \leq x<b .
$$

The following result provides sufficient conditions for the convexity and concavity of ${ }^{c} D_{a}^{\alpha} f($ see $[13])$.

Lemma 7. Let $0<\alpha<1$ and $f \in C^{3}([a, b] ; \mathbb{R})$. If $f^{\prime \prime \prime}(x) \geq 0$ in $[a, b]$, $f^{\prime \prime}(a) \leq 0$ and $f^{\prime}(a) \geq 0$, then ${ }^{c} D_{a}^{\alpha} f$ is concave in $[a, b]$. If $f^{\prime \prime \prime}(x) \leq 0$ in $[a, b], f^{\prime \prime}(a) \geq 0$ and $f^{\prime}(a) \geq 0$, then ${ }^{c} D_{a}^{\alpha} f$ is convex in $[a, b]$.

Using Lemma 7, we deduce the following criteria for the convexity and concavity of $\left|{ }^{c} D_{a}^{\alpha} f\right|$.

Lemma 8. Let $0<\alpha<1$ and $f \in C^{3}([a, b] ; \mathbb{R})$ be such that

$$
f^{\prime}(x) \geq 0, \quad a \leq x \leq b
$$

If $f^{\prime \prime \prime}(x) \geq 0$ in $[a, b]$ and $f^{\prime \prime}(a) \leq 0$, then $\left|{ }^{c} D_{a}^{\alpha} f\right|$ is concave in $[a, b]$. If $f^{\prime \prime \prime}(x) \leq 0$ in $[a, b]$ and $f^{\prime \prime}(a) \geq 0$, then $\left|{ }^{c} D_{a}^{\alpha} f\right|$ is convex in $[a, b]$.

Proof. We have just to observe that, by (7), we have

$$
\left({ }^{c} D_{a}^{\alpha} f\right)(x) \geq 0, \quad a \leq x \leq b .
$$

Next, using Lemma 7, the desired results follow.

\section{Results and Discussion}

In this section, we state and prove our main results. Just before, let us fix $0<\alpha<1$ and $a, b \in \mathbb{R}$ with $a<b$.

First, we shall establish the following fractional version of Lemma 1.

Lemma 9. Let $f \in C^{1}([a, b] ; \mathbb{R})$. Then,

$$
\frac{b-a}{2} \int_{0}^{1}(1-2 t)\left({ }^{c} D_{a}^{\alpha} f\right)(t a+(1-t) b) d t=\left[\frac{\alpha f(a)(b-a)^{1-\alpha}}{2 \Gamma(3-\alpha)}+\frac{1}{2}\left(I_{a}^{1-\alpha} f\right)(b)\right]-\frac{1}{b-a}\left(I_{a}^{2-\alpha} f\right)(b) .
$$

Proof. By the definition of the left-side Liouville-Caputo fractional derivative of order $\alpha$, we have

$$
\int_{a}^{b}\left(x-\frac{a+b}{2}\right)\left({ }^{c} D_{a}^{\alpha} f\right)(x) d x=\int_{a}^{b}\left(x-\frac{a+b}{2}\right)\left(I_{a}^{1-\alpha} f^{\prime}\right)(x) d x .
$$

Using the integration by parts rule given by Lemma 4, we obtain

$$
\int_{a}^{b}\left(x-\frac{a+b}{2}\right)\left({ }^{c} D_{a}^{\alpha} f\right)(x) d x=\int_{a}^{b} f^{\prime}(x) I_{b}^{1-\alpha}\left(x-\frac{a+b}{2}\right) d x .
$$

Next, the standard integration by parts rule yields

$$
\int_{a}^{b}\left(x-\frac{a+b}{2}\right)\left({ }^{c} D_{a}^{\alpha} f\right)(x) d x=\left[f(x) I_{b}^{1-\alpha}\left(x-\frac{a+b}{2}\right)\right]_{x=a}^{b}-\int_{a}^{b} f(x) \frac{d}{d x} I_{b}^{1-\alpha}\left(x-\frac{a+b}{2}\right) d x .
$$


On the other hand, by the definition of the right-side Riemann-Liouville fractional integral of order $1-\alpha$, we have

$$
\begin{aligned}
& I_{b}^{1-\alpha}\left(x-\frac{a+b}{2}\right) \\
& =\frac{1}{\Gamma(1-\alpha)} \int_{x}^{b}(t-x)^{-\alpha}\left(t-\frac{a+b}{2}\right) d t \\
& =\frac{1}{\Gamma(1-\alpha)}\left(\int_{x}^{b} t(t-x)^{-\alpha} d t-\frac{a+b}{2} \int_{x}^{b}(t-x)^{-\alpha} d t\right) \\
& =\frac{1}{\Gamma(1-\alpha)}\left(\frac{1}{1-\alpha}\left[t(t-x)^{1-\alpha}\right]_{t=x}^{b}-\frac{1}{1-\alpha} \int_{x}^{b}(t-x)^{1-\alpha} d t-\frac{(a+b)}{2(1-\alpha)}(b-x)^{1-\alpha}\right) \\
& =\frac{1}{\Gamma(1-\alpha)}\left(\frac{b(b-x)^{1-\alpha}}{1-\alpha}-\frac{(b-x)^{2-\alpha}}{(1-\alpha)(2-\alpha)}-\frac{(a+b)}{2(1-\alpha)}(b-x)^{1-\alpha}\right) .
\end{aligned}
$$

Using the identity

$$
s \Gamma(s)=\Gamma(s+1), \quad s>0,
$$

we obtain

$$
I_{b}^{1-\alpha}\left(x-\frac{a+b}{2}\right)=\frac{(b-x)^{1-\alpha}}{\Gamma(2-\alpha)}\left(\frac{b-a}{2}-\frac{b-x}{2-\alpha}\right),
$$

which yields

$$
\left[f(x) I_{b}^{1-\alpha}\left(x-\frac{a+b}{2}\right)\right]_{x=a}^{b}=\frac{\alpha f(a)(b-a)^{2-\alpha}}{2 \Gamma(3-\alpha)}
$$

and

$$
\frac{d}{d x} I_{b}^{1-\alpha}\left(x-\frac{a+b}{2}\right)=\frac{(b-x)^{1-\alpha}}{\Gamma(2-\alpha)}-\left(\frac{b-a}{2}\right) \frac{(b-x)^{-\alpha}}{\Gamma(1-\alpha)} .
$$

Using (11), we obtain

$$
\begin{aligned}
& \int_{a}^{b} f(x) \frac{d}{d x} I_{b}^{1-\alpha}\left(x-\frac{a+b}{2}\right) d x \\
& =\frac{1}{\Gamma(2-\alpha)} \int_{a}^{b}(b-x)^{1-\alpha} f(x) d x-\left(\frac{b-a}{2}\right) \frac{1}{\Gamma(1-\alpha)} \int_{a}^{b}(b-x)^{-\alpha} f(x) d x
\end{aligned}
$$

i.e.,

$$
\int_{a}^{b} f(x) \frac{d}{d x} I_{b}^{1-\alpha}\left(x-\frac{a+b}{2}\right) d x=\left(I_{a}^{2-\alpha} f\right)(b)-\left(\frac{b-a}{2}\right)\left(I_{a}^{1-\alpha} f\right)(b) .
$$

Next, combining (9), (10) and (12), we obtain

$$
\frac{1}{b-a} \int_{a}^{b}\left(x-\frac{a+b}{2}\right)\left({ }^{c} D_{a}^{\alpha} f\right)(x) d x=\left[\frac{\alpha f(a)(b-a)^{1-\alpha}}{2 \Gamma(3-\alpha)}+\frac{1}{2}\left(I_{a}^{1-\alpha} f\right)(b)\right]-\frac{1}{b-a}\left(I_{a}^{2-\alpha} f\right)(b) .
$$

Finally, the change of variable

$$
x=t a+(1-t) b, \quad 0 \leq t \leq 1,
$$

yields (8).

Remark 1. Passing to the limit as $\alpha \rightarrow 1^{-}$in (8) and using Lemmas 3 and 6 , we obtain (6).

Using a similar argument as in the proof of Lemma 9, we obtain the following fractional version of Lemma 1. 
Lemma 10. Let $f \in C^{1}([a, b] ; \mathbb{R})$. Then,

$$
\frac{b-a}{2} \int_{0}^{1}(1-2 t)\left(-{ }^{c} D_{b}^{\alpha} f\right)(t a+(1-t) b) d t=\left[\frac{\alpha f(b)(b-a)^{1-\alpha}}{2 \Gamma(3-\alpha)}+\frac{1}{2}\left(I_{b}^{1-\alpha} f\right)(a)\right]-\frac{1}{b-a}\left(I_{b}^{2-\alpha} f\right)(a) .
$$

Remark 2. Passing to the limit as $\alpha \rightarrow 1^{-}$in (13) and using Lemmas 3 and 6, we obtain (6).

Our first main result is the following fractional version of Theorem 1.

Theorem 3. Let $f \in \operatorname{Conv}_{a}^{\alpha}([a, b] ; \mathbb{R})$, where $\operatorname{Conv}_{a}^{\alpha}([a, b] ; \mathbb{R})$ is the class of functions given by (1). Then,

$$
\left|\left[\frac{\alpha f(a)(b-a)^{1-\alpha}}{2 \Gamma(3-\alpha)}+\frac{1}{2}\left(I_{a}^{1-\alpha} f\right)(b)\right]-\frac{1}{b-a}\left(I_{a}^{2-\alpha} f\right)(b)\right| \leq \frac{(b-a)}{8}\left|\left({ }^{c} D_{a}^{\alpha} f\right)(b)\right| .
$$

Proof. Let $f \in \operatorname{Conv}_{a}^{\alpha}([a, b] ; \mathbb{R})$. Using Lemma 9, we obtain

$$
\begin{aligned}
& \left|\left[\frac{\alpha f(a)(b-a)^{1-\alpha}}{2 \Gamma(3-\alpha)}+\frac{1}{2}\left(I_{a}^{1-\alpha} f\right)(b)\right]-\frac{1}{b-a}\left(I_{a}^{2-\alpha} f\right)(b)\right| \\
& \leq \frac{b-a}{2} \int_{0}^{1}|1-2 t|\left|{ }^{c} D_{a}^{\alpha} f\right|(t a+(1-t) b) d t .
\end{aligned}
$$

On the other hand, using the convexity of $\left|{ }^{c} D_{a}^{\alpha} f\right|$, we obtain

$$
\begin{aligned}
& \frac{b-a}{2} \int_{0}^{1}|1-2 t|\left|{ }^{c} D_{a}^{\alpha} f\right|(t a+(1-t) b) d t \\
& \leq \frac{b-a}{2}\left(\left|\left({ }^{c} D_{a}^{\alpha} f\right)(a)\right| \int_{0}^{1}|1-2 t| t d t+\left|\left({ }^{c} D_{a}^{\alpha} f\right)(b)\right| \int_{0}^{1}|1-2 t|(1-t) d t\right) .
\end{aligned}
$$

Using the fact that $\left({ }^{c} D_{a}^{\alpha} f\right)(a)=0$ and

$$
\int_{0}^{1}|1-2 t|(1-t) d t=\frac{1}{4}
$$

we obtain

$$
\begin{aligned}
& \frac{b-a}{2} \int_{0}^{1}|1-2 t|\left|{ }^{c} D_{a}^{\alpha} f\right|(t a+(1-t) b) d t \\
& \leq \frac{(b-a)}{8}\left|\left({ }^{c} D_{a}^{\alpha} f\right)(b)\right| .
\end{aligned}
$$

Finally, combining (15) and (16), we obtain (14).

Next, we discuss the case when $f \in \operatorname{Conv}_{b}^{\alpha}([a, b] ; \mathbb{R})$, where $\operatorname{Conv}_{b}^{\alpha}([a, b] ; \mathbb{R})$ is the class of functions given by (2).

Theorem 4. Let $f \in \operatorname{Conv}_{b}^{\alpha}([a, b] ; \mathbb{R})$. Then,

$$
\left|\left[\frac{\alpha f(b)(b-a)^{1-\alpha}}{2 \Gamma(3-\alpha)}+\frac{1}{2}\left(I_{b}^{1-\alpha} f\right)(a)\right]-\frac{1}{b-a}\left(I_{b}^{2-\alpha} f\right)(a)\right| \leq \frac{(b-a)}{8}\left|\left({ }^{c} D_{b}^{\alpha} f\right)(a)\right| .
$$

Proof. Using Lemma 10, the convexity of $\left|{ }^{c} D_{b}^{\alpha} f\right|$ and a similar argument as in the proof of Theorem 3 , we obtain (17).

Furthermore, we consider the case when $f \in \operatorname{Conc}_{a}^{\alpha}([a, b] ; \mathbb{R})$, where $\operatorname{Conc}_{a}^{\alpha}([a, b] ; \mathbb{R})$ is the class of functions given by (3). We obtain the following fractional version of Theorem 2. 
Theorem 5. Let $f \in \operatorname{Conc}_{a}^{\alpha}([a, b] ; \mathbb{R})$. Then,

$$
\left|\left[\frac{\alpha f(a)(b-a)^{1-\alpha}}{2 \Gamma(3-\alpha)}+\frac{1}{2}\left(I_{a}^{1-\alpha} f\right)(b)\right]-\frac{1}{b-a}\left(I_{a}^{2-\alpha} f\right)(b)\right| \leq \frac{(b-a)}{4}\left|\left({ }^{c} D_{a}^{\alpha} f\right)\left(\frac{a+b}{2}\right)\right| .
$$

Proof. Using the concavity of $\left|{ }^{c} D_{a}^{\alpha} f\right|$ and Jensen integral inequality, we obtain

$$
\begin{aligned}
& \frac{b-a}{2} \int_{0}^{1}|1-2 t|\left|{ }^{c} D_{a}^{\alpha} f\right|(t a+(1-t) b) d t \\
& \leq \frac{b-a}{2}\left(\int_{0}^{1}|1-2 t| d t\right)\left|{ }^{c} D_{a}^{\alpha} f\right|\left(\frac{\int_{0}^{1}|1-2 t|(t a+(1-t) b) d t}{\int_{0}^{1}|1-2 t| d t}\right) \\
& =\frac{(b-a)}{4}\left|\left({ }^{c} D_{a}^{\alpha} f\right)\left(\frac{a+b}{2}\right)\right| .
\end{aligned}
$$

Using Lemma 9 and the above inequality, (18) follows.

Using a similar argument as in the proof of Theorem 5 , we obtain the following result concerning the case when $f \in \operatorname{Conc}_{b}^{\alpha}([a, b] ; \mathbb{R})$, where $\operatorname{Conc}_{b}^{\alpha}([a, b] ; \mathbb{R})$ is the class of functions given by (4).

Theorem 6. Let $f \in \operatorname{Conc}_{b}^{\alpha}([a, b] ; \mathbb{R})$. Then,

$$
\left|\left[\frac{\alpha f(b)(b-a)^{1-\alpha}}{2 \Gamma(3-\alpha)}+\frac{1}{2}\left(I_{b}^{1-\alpha} f\right)(a)\right]-\frac{1}{b-a}\left(I_{b}^{2-\alpha} f\right)(a)\right| \leq \frac{(b-a)}{4}\left|\left({ }^{c} D_{b}^{\alpha} f\right)\left(\frac{a+b}{2}\right)\right| .
$$

Remark 3. Let us consider the classes of functions

$$
\operatorname{Conv}^{1}([a, b] ; \mathbb{R})=\left\{f \in C^{1}([a, b] ; \mathbb{R}):\left|f^{\prime}\right| \text { is convex }\right\} .
$$

Observe that

$$
\operatorname{Conv}^{1}([a, b] ; \mathbb{R}) \nsubseteq \operatorname{Conv}_{a}^{\alpha}([a, b] ; \mathbb{R}) .
$$

To show this, let us consider as example the function

$$
f(x)=(x-a)^{\beta}, \quad a \leq x \leq b,
$$

where

$$
\alpha+1 \leq \beta<2 .
$$

We have $f \in C^{1}([a, b] ; \mathbb{R})$. Moreover,

$$
\left|f^{\prime}(x)\right|=\beta(x-a)^{\beta-1}, \quad a \leq x \leq b .
$$

It can be easily seen that $\left|f^{\prime}\right|$ is concave (so, nonconvex). On the other hand, we have

$$
\left|\left({ }^{c} D_{a}^{\alpha} f\right)(x)\right|=\frac{\Gamma(\beta+1)}{\Gamma(\beta+1-\alpha)}(x-a)^{\beta-\alpha}, \quad a \leq x \leq b,
$$

which is a convex function in $[a, b]$. Therefore, $f \in \operatorname{Conv}_{a}^{\alpha}([a, b] ; \mathbb{R})$ but $f \notin \operatorname{Conv}^{1}([a, b] ; \mathbb{R})$. Hence, Theorem 3 is a real extension of Theorem 1.

\section{Applications to Special Means of Real Numbers}

In this section, we provide some applications to special means of real numbers. Let

$$
A(u, v)=\frac{u+v}{2}, \quad u, v \in \mathbb{R}
$$


The quantity $A(u, v)$ is known in the literature as the arithmetic mean of $u$ and $v$. Let

$$
L_{\sigma}(u, v)=\left[\frac{v^{\sigma+1}-u^{\sigma+1}}{(\sigma+1)(v-u)}\right]^{\frac{1}{\sigma}}, \quad \sigma>-1, \sigma \neq 0,0<u<v .
$$

The quantity $L_{\sigma}(u, v)$ can be considered as a fractional generalized ln-mean of $u$ and $v$. We have the following estimate.

Corollary 1. Let $1 \leq \beta \leq 2$ and $0<a<b$. Then,

$$
\left|A\left(a^{\beta}, b^{\beta}\right)-\left[L_{\beta}(a, b)\right]^{\beta}\right| \leq \frac{\beta(b-a) b^{\beta-1}}{8} .
$$

Proof. Let $1 \leq \beta \leq 2$ and $0<a<b$. Let us consider the function

$$
f(x)=x^{\beta}, \quad a \leq x \leq b .
$$

Using Lemma 8, we deduce that $\left|{ }^{c} D_{a}^{\alpha} f\right|$ is convex in $[a, b]$, for every $0<\alpha<1$. Hence,

$$
f \in \operatorname{Conv}_{a}^{\alpha}([a, b] ; \mathbb{R}), \quad 0<\alpha<1 .
$$

Therefore, using Theorem 3 , for all $0<\alpha<1$, we obtain

$$
\left|\left[\frac{\alpha a^{\beta}(b-a)^{1-\alpha}}{2 \Gamma(3-\alpha)}+\frac{1}{2}\left(I_{a}^{1-\alpha} f\right)(b)\right]-\frac{1}{b-a}\left(I_{a}^{2-\alpha} f\right)(b)\right| \leq \frac{(b-a)}{8}\left|\left({ }^{c} D_{a}^{\alpha} f\right)(b)\right| .
$$

Passing to the limit as $\alpha \rightarrow 1^{-}$in (21) and using Lemmas 3 and 6, we obtain (19).

Remark 4. Let us consider the case $1<\beta<2$. In this case, by (ii) and (iv), we have $\left|f^{\prime}\right|$ is concave in $[a, b]$, where $f$ is the function given by (20). Therefore, Theorem 1 cannot be applied. On the other hand, by Theorem 2, we have

$$
\left|\frac{f(a)+f(b)}{2}-\frac{1}{b-a} \int_{a}^{b} f(x) d x\right| \leq \frac{b-a}{4}\left|f^{\prime}\left(\frac{a+b}{2}\right)\right|,
$$

i.e.,

$$
\left|A\left(a^{\beta}, b^{\beta}\right)-\left[L_{\beta}(a, b)\right]^{\beta}\right| \leq \frac{\beta(b-a)}{4}\left(\frac{a+b}{2}\right)^{\beta-1} .
$$

Observe that

$$
\frac{\beta(b-a) b^{\beta-1}}{8}<\frac{\beta(b-a)}{4}\left(\frac{a+b}{2}\right)^{\beta-1} .
$$

Therefore, the estimate (19) is sharper than (22).

Remark 5. Let us consider the case $\beta=2$. In this case, $\left|f^{\prime}\right|$ is convex in $[a, b]$, where $f$ is the function given by (20) $\left(f(x)=x^{2}\right)$. Therefore, by Theorem 1, we have

$$
\left|A\left(a^{2}, b^{2}\right)-\left[L_{2}(a, b)\right]^{2}\right| \leq \frac{b^{2}-a^{2}}{4} .
$$

On the other hand, taking $\beta=2$ in (19), we obtain

$$
\left|A\left(a^{2}, b^{2}\right)-\left[L_{2}(a, b)\right]^{2}\right| \leq \frac{(b-a) b}{4} .
$$

Observe that

$$
\frac{(b-a) b}{4}<\frac{b^{2}-a^{2}}{4} .
$$


Therefore, (24) is sharper than (23).

Author Contributions: All authors contributed equally in writing this article. All authors read and approved the final manuscript.

Funding: This research received no external funding.

Conflicts of Interest: The authors declare no conflict of interest.

\section{References}

1. Beckenbach, E.F. Convex functions. Bull. Am. Math. Soc. 1948, 54, 439-460.

2. Mitrinović, D.S.; Lacković, I.B. Hermite and convexity. Aequ. Math. 1985, 28, 229-232. [CrossRef]

3. Bombardelli, M.; Varošanec, S. Properties of h-convex functions related to the Hermite-Hadamard-Fejér inequalities. Comput. Math. Appl. 2009, 58, 1869-1877.

4. Dragomir, S.S.; Pearce, C.E.M. Quasi-convex functions and Hadamard's inequality. Bull. Aust. Math. Soc. 1998, 57, 377-385.

5. Farissi El, A. Simple proof and refeinment of Hermite-Hadamard inequality. J. Math. Inequal. 2010, 4, 365-369.

6. Rajba, T. On strong delta-convexity and Hermite-Hadamard type inequalities for delta-convex functions of higher order. Math. Inequal. Appl. 2015, 18, 267-293.

7. Samet, B. On an implicit convexity concept and some integral inequalities. J. Inequal. Appl. 2016. [CrossRef]

8. Sarikaya, M.Z.; Saglam, A.; Yildirim, H. On some Hadamard-type inequalities for h-convex functions. J. Math. Inequal. 2008, 2, 335-341.

9. Dragomir, S.S.; Agarwal, R.P. Two inequities for differentiable mappings and applications to special means of real numbers and to trapezoidal formula. Appl. Math. Lett. 1998, 11, 91-95.

10. Pearce, C.E.M.; Pečarić, J. Inequalities for differentiable mappings with application to special means and quadrature formulm. Appl. Math. Lett. 2000, 13, 51-55.

11. Almeida, R. A Caputo fractional derivative of a function with respect to another function. Commun. Nonlinear Sci. Numer. Simul. 2017, 44, 460-481.

12. Kilbas, A.A.; Srivastava, H.M.; Trujillo, J.J. Theory and applications of fractional differential equations. In North-Holland Mathematics Studies; Elsevier Science B.V.: Amsterdam, The Netherlands, 2006; Volume 204.

13. Zhou, X.F.; Liu, S.; Zhang, Z.; Jiang, W. Monotonicity, Concavity, and Convexity of Fractional Derivative of Functions. Sci. World J. 2013, 2013. [CrossRef]

(C) 2018 by the authors. Licensee MDPI, Basel, Switzerland. This article is an open access article distributed under the terms and conditions of the Creative Commons Attribution (CC BY) license (http://creativecommons.org/licenses/by/4.0/). 